\title{
Zinc oxide for electronic, photovoltaic and optoelectronic applications
}

\author{
M. Godlewski ${ }^{1,2}$, E. Guziewicz ${ }^{1}$, K. Kopalko ${ }^{1}$, G. Łuka ${ }^{1}$, M.I. Łukasiewicz ${ }^{1}$, \\ T. Krajewski ${ }^{1}$, B.S. Witkowski ${ }^{1}$, and S. Gieraltowska ${ }^{1}$ \\ ${ }^{1}$ Institute of Physics, Polish Academy of Sciences, Al. Lotników 32/46, Warsaw, Poland \\ ${ }^{2}$ Department of Mathematics and Natural Sciences College of Science, \\ Cardinal Stefan Wyszyński University, Warsaw, Poland \\ E-mail: godlew@ifpan.edu.p
}

Received September 24, 2010

\begin{abstract}
We demonstrate that the atomic layer deposition (ALD) technique has large potential to be widely used in a production of $\mathrm{ZnO}$ films for applications in electronic, photovoltaic (PV) and optoelectronic devices. Low growth temperature makes the ALD-grown $\mathrm{ZnO}$ films suitable for construction of various semiconductor/organic material hybrid structures. This opens possibilities of construction of novel devices based on very cheap organic materials. This includes organic light emitting diodes and PV cells of the third generation, as discussed in the present work.
\end{abstract}

PACS: $81.05 . \mathrm{Dz}$ II-VI semiconductors;

85.40.Xx Hybrid microelectronics; thick films;

75.50.Pp Magnetic semiconductors;

81.05.Fb Organic semiconductors;

88.40.jm Thin film III-V and II-VI based solar cells;

88.40.jr Organic photovoltaics.

Keywords: $\mathrm{ZnO}$ films, semiconductor/organic material hybrid structures, applications of $\mathrm{ZnO}$.

\section{Introduction}

At present electronics is based mostly on silicon, whereas other materials, such as GaAs or GaN, are used only in very specialized devices. The dominant role of silicon was not questioned till very recently, until the necessity to go to a 3D architecture became evident. Such architecture allows further increase of capacity of integrated circuits. The research on 3D-like devices is the most advanced in the case of nonvolatile memories. One of the materials studied for a new generation of so-called cross-bar memories with a $3 \mathrm{D}$ architecture is $\mathrm{ZnO}$ [1], as we discuss below. This and other possible use of $\mathrm{ZnO}$ in electronic devices will be discussed in the present work.

Considering other possible applications of $\mathrm{ZnO}$, the one discussed below clearly cannot be based on silicon. We mean here transparent electronic devices. For them use of $\mathrm{ZnO}$ is quite obvious. Due to a large band gap of $\mathrm{ZnO}$ this material is transparent to the visible light.

It seemed at first that $\mathrm{ZnO}$ will make career in optoelectronic devices similar to that of $\mathrm{GaN}$ and its alloys. This did not happened till now since stable $p$-type doping of
$\mathrm{ZnO}$ was not realized yet. However, new applications of $\mathrm{ZnO}$ in optoelectronic devices are still possible, as also discussed in the present paper.

Finally, a breakthrough in construction of photovoltaic (PV) devices is required to allow for economically justified wide use of a solar energy. There are several indications that $\mathrm{ZnO}$ can play an important role also in this area of electronic industry. We present here some examples of use of $\mathrm{ZnO}$ in PV devices.

\section{ZnO films for electronic applications}

\section{1. $\mathrm{ZnO}$ for $3 \mathrm{D}$ memory devices}

In Autumn of 2007 we witnessed a new revolution in a structure of integrated circuits (IC). In ICs introduced by the Intel company $\mathrm{SiO}_{2}$ was replaced by $\mathrm{HfO}_{2}$ as a gate isolator. This enabled introduction of the ICs with $45 \mathrm{~nm}$ node architecture (Autumn of 2007) and $32 \mathrm{~nm}$ node (Spring of 2010). For these devices a physical size of field effect transistor was scaled down to about $100 \mathrm{~nm}$. 
The ICs with the $22 \mathrm{~nm}$ node architecture are predicted to be introduced within next two-three years. Further scaling down of transistor sizes may however be difficult. Thus, further increase of capacity of electronic devices may be due to introduction of a new architecture. The most promising one is the 3D-like, with active elements stacked one over another. The research on such devices is very advanced in the case of so-called cross-bar memories [1], which are shortly described below.

At present, the nonvolatile semiconductor-based random access memories (RAM) use architecture of a floating gate. It is very close to the one of a field effect transistor. The only difference is the extra gate embedded between isolating films. This similarity means that as in the case of ICs miniaturization limit will soon be achieved and that we need another approach to increase further a memory storage capacity. One of the most studied ideas here is based on vertically stacked memory cells (cross-bar memory) (see, e.g., [1-3]). The storage density of such memory devices depends on $4 F^{2} / n$ ratio, where $F$ is a node standard (e.g., $65 \mathrm{~nm}$ ) and $n$ number of stacks. The smaller this ratio is, the higher density can be achieved. We need $n=3$ or more to surpass floating gate memories in a memory storage capacity.

Unfortunately, construction of 3D electronic devices imposes serious material and processing restrictions, and thus must result in serious changes in device processing. In 3D structures, with active parts stacked one over other, metal paths will not be on top of the devices, but both beneath and above electronic elements. This drastically restricts growth temperature to below $350-400{ }^{\circ} \mathrm{C}[1,2]$. For higher temperatures metal paths will be affected. The necessity of temperature reduction means that the dominant role of silicon in memory devices is questioned for the first time from many years.

In the cross-bar memory architecture a single element of the structure consists of a so-called selector (allowing addressing of a single cell) and a storage element. By a selector we mean here either a Schottky or $p-n$ junction $[1,3]$. The selector must be characterized by a very high rectification factor to block cross talk between cells. Moreover, it must allow a high current density, necessary to address NiO [3] or phase change memory (PCM) materials, which likely will be used as information storage elements.

One of the materials considered for cross-bar memory applications is $\mathrm{ZnO}$ [1]. In fact, we demonstrate here that $\mathrm{ZnO}$ can be material of choice for the cross-bar memories $[1,2]$. To produce $\mathrm{ZnO}$-based junctions with appropriate electrical parameters we used the growth technique of atomic layer deposition (ALD). As zinc precursor we used diethylzinc (DEZn) with deionized water used as an oxygen precursor $[1,2,4-6]$ :

$$
\mathrm{Zn}\left(\mathrm{C}_{2} \mathrm{H}_{5}\right)_{2}+\mathrm{H}_{2} \mathrm{O} \rightarrow \mathrm{ZnO}+2 \mathrm{C}_{2} \mathrm{H}_{6} .
$$

Use of the ALD and DEZn enabled us reduction of the growth temperature to about $100{ }^{\circ} \mathrm{C}$, which enabled not only construction of Schottky diodes with excellent rectification rates [1,3], but also fully $\mathrm{ZnO}$-based $p-n$ diodes [6]. Moreover, low growth temperature allowed us construction of hybrid structures of the type $\mathrm{ZnO} /$ organic material $[1,7-9]$ with very good rectification rate and high current density, as will be discussed further on.

The record high rectification of about $10^{8}$ was achieved by us for Schottky structures with silver used as a Schottky contact, but only when deposited below the $\mathrm{ZnO}$ film $[1,3]$.

\subsection{ZnO for transparent electronic devices}

$\mathrm{ZnO}$ is an ideal material for the application in the transparent electronics [6]. The required electron concentration in the range of $10^{17}-10^{18} \mathrm{~cm}^{-3}$ can be achieved [6]. Importantly, we observed that electron mobility in $\mathrm{ZnO}$ films grown by the ALD is relatively weakly dependent on film crystallinity $[10,11]$. For example, for our polycrystalline $\mathrm{ZnO}$ films grown at low temperature on glass substrate electron mobility is between 5 and $150 \mathrm{~cm}^{2} /(\mathrm{V} \cdot \mathrm{s})$ at room temperature (RT) $[4,10,11]$. Moreover, the ALD enables growth of $\mathrm{ZnO}$ on polymers, plastic foil, glass indium tin oxide (ITO) (see Fig. 1 in which we show model of field effect transistors grown and studied by us), etc... crucial for its application in transparent electronics. As the gate oxide (dielectric) thin films of $\mathrm{Al}_{2} \mathrm{O}_{3}, \mathrm{HfO}_{2}$ (see Fig. 2), or their laminar structure can be used. Also these layers we deposit by the ALD [12].

\section{3. $\mathrm{ZnO}$ for spintronic applications}

Spintronics is a booming field of electronics, with range of devices which are commercialized. These devices are

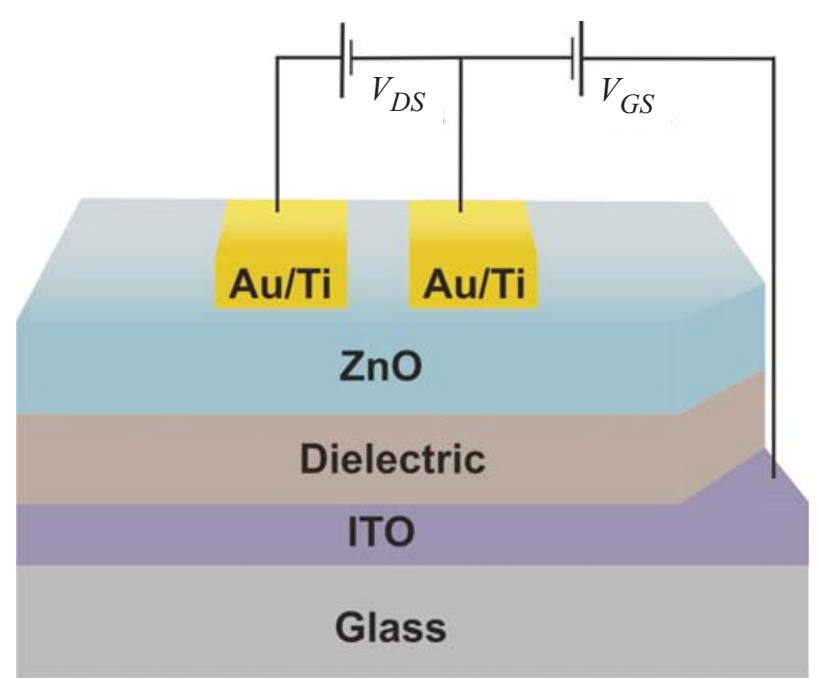

Fig. 1. Model of transparent field effect transistor based on the ALD grown thin films of dielectric (we use $\mathrm{Al}_{2} \mathrm{O}_{3}, \mathrm{HfO}_{2}$ or their laminar structure) and $\mathrm{ZnO}$ layers. 


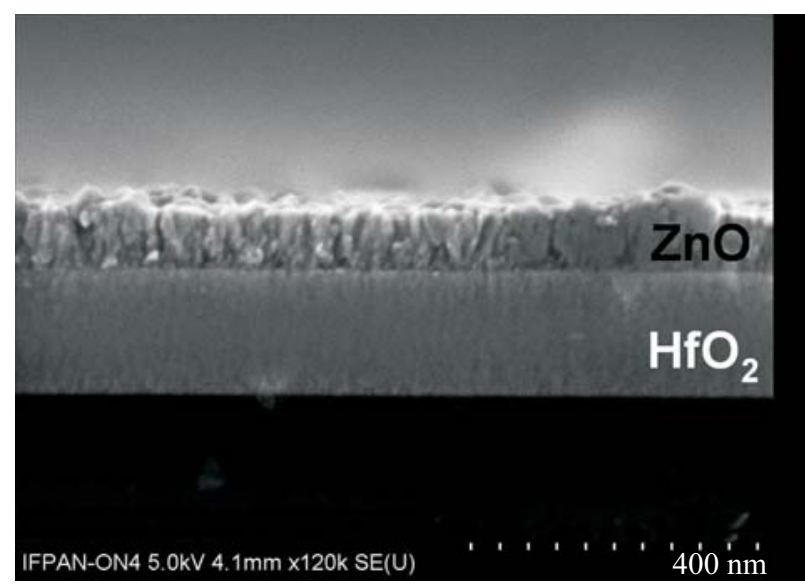

Fig. 2. Cross-section of dielectric/ZnO thin film structure deposited by the ALD as a part of transparent transistor taken with scanning electron microscope.

based on GMR or TMR concept (giant magneto-resistivity or tunneling magneto-resistivity, respectively). At present, the research concentrates on introduction of the most futuristic spintronic device (so-called spin transistor [13]), which will use spin of an electron rather than its charge to functionalize operation of metal-oxide-semiconductor (MOS) transistors. It is expected that manipulation of electron's spin (or resulting magnetism) rather than its charge will help to achieve new or improved (faster devices working at lower energy consumption) functionalities of such devices.

The concept of the spin transistor was proposed by Datta and Das [13]. Spin transistor consists of a spin injector, conventional semiconductor with a gate to manipulate electrons spin (via the Rashba effect) and as a spin analyzer (used to check the resulting spin orientation of electrons).

The spin transistor is, however, very difficult to be realized in the practice. Only very recently Toshiba Company announced realization of such transistor with a full-Heusler alloy used for both the spin injector and analyzer. It is still believed that to achieve advantages of the spin transistor we need to use semiconductor-based material for the spin injector and analyzer. It will be so-called diluted magnetic semiconductor (DMS) showing ferromagnetic (FM) phase at RT. The 20 years delay in the introduction of the spin transistor relates to the lack of DMS materials ferromagnetic at the RT.

III-Mn-V semiconductors were the most studied for applications in spintronic devices. However, the failure to reach RT FM for GaMnAs, the most studied diluted magnetic semiconductor, moved the interest to other materials. At present, $\mathrm{ZnO}$ and $\mathrm{GaN}$ are the most studied DMS materials. After the theoretical prediction of above RT FM of heavily $p$-type GaMnN and $\mathrm{ZnMnO}$ [14] more than 1000 papers were published on magnetic properties of these two wide band gap semiconductors doped with transition metal
(TM) ions. More than $50 \%$ of them were on $\mathrm{ZnO}$, becoming the most studied material recently. This partly relates to the first observation of above RT FM in $\mathrm{ZnCoO}$ [15], followed by many other similar reports.

The present situation is very confusing. Reproducibility of the results is low, reports contradict each other, etc. Moreover, the theoretical report on RT FM of $\mathrm{ZnMnO}$ and $\mathrm{GaMnN}$ assumed 2+ charge state of $\mathrm{Mn}$ in heavily $p$-type $\mathrm{ZnO}$ or GaN. This, however, cannot be realized experimentally. In both these materials Mn recharges to $3+$ $[16,17]$ in $p$-type samples.

It is now the widely accepted statement that RT FM is not the material property of $\mathrm{ZnO}$ (also $\mathrm{GaN}$ ), but relates to some local properties like doping nonuniformities. Our detail studies of $\mathrm{ZnMnO}$ clearly indicate that once TM is distributed uniformly $\mathrm{ZnMnO}$ is paramagnetic $[18,19]$.

To get uniform TM distribution we applied technique of the ALD and a low growth temperature [19]. Low growth temperature turned out to be important also in the case of $\mathrm{ZnMnO}$ nanoparticles [20]. Once samples were grown at increased temperature, or we used post-growth annealing, TM rich regions appear. These regions can be clearly de-
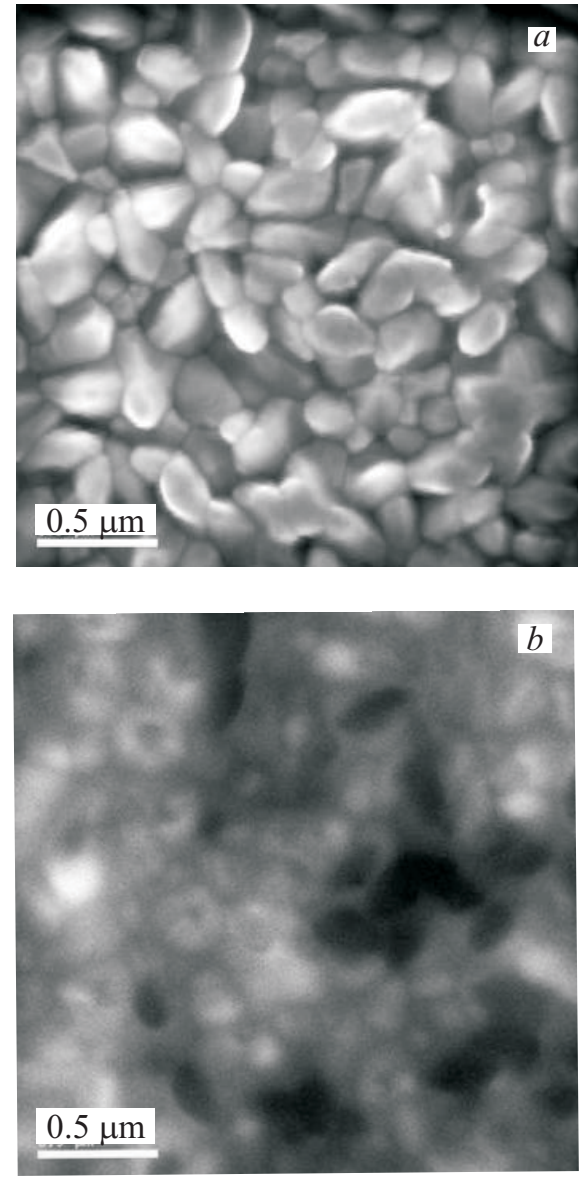

Fig. 3. Scanning electron microscopy image (a) and cathodoluminescence $(b)$ map (with detection set at the band edge emission) taken at $10 \mathrm{kV}$ from $2 \times 2 \mu \mathrm{m}$ region of $\mathrm{ZnCoO}$ films grown at low temperature by the ALD and then annealed at $800{ }^{\circ} \mathrm{C}$. 
picted using cathodoluminescence (CL) [21,22]. In the CL study we utilized the fact that TM ions, such as $\mathrm{Mn}$ or Co, effectively quench visible emission of $\mathrm{ZnO}[21,22]$. In the consequence TM rich regions are observed in the CL study as dark spots (see Fig. 3). Such spots are observed in samples with nonuniform Co distribution (after annealing at $800{ }^{\circ} \mathrm{C}$ ). In as-grown uniform films dark area are not observed [22]. Applying depth-profiling CL (see [23] for the explanation of the method) we proved that such TM rich regions penetrate whole structure in the form of columns.

As already mentioned RT FM of $\mathrm{ZnCoO}$ was reported already in 2001 [15]. Despite nearly 10 years of concentrated research the origin of the RT FM is still not clear [24]. The presence of RT FM was related to intrinsic lattice defects of $\mathrm{ZnO}$ [25], foreign phases in the form of various TM oxides (e.g., CoO [26]), metal accumulations [27], unpaired spins at TM rich regions [28,29], etc.

The most puzzling situation relates to the role of deep lattice defects (vacancies?) in FM of ZnTMO [30-33]. This further complicates situation since our CL investigations indicate that high temperature annealing leading in the appearance of FM response not only results in nonuniformities of Co $(\mathrm{Mn})$ distribution, but also in the generation of deep lattice defects, as we observed in the CL investigations (see Fig. 4). Figure 4 shows CL spectra taken at four different accelerating voltages (depth profiling) for as-grown $\mathrm{ZnCoO}$ film (with very uniform Co distribution) and after annealing the film at 400,600 and $800{ }^{\circ} \mathrm{C}$. The latter sample was nonuniform. Such films show FM signal. Deep defects, responsible for defect-related emissions at longer wavelength, are generated mostly at the interface region, as deduced from the depth-profiling CL investigations, as shown in Fig. 4.

Regarding the defects, hydrogen can also be important in the formation of FM coupled TM pairs. Park and Chadi [34] proposed that hydrogen may interact with TM ions resulting in the FM phase. Bridge-like structures $\mathrm{Co}-\mathrm{H}-\mathrm{Co}$ may be formed, in which spins of Co ions are aligned parallel. In fact some our recent investigations [35] support such possibility. This observation indicates that the mechanism responsible for the FM response can depend on growth details and is different from sample to sample.

For application in devices such as the spin transistor it remains important if we can achieve significant spin polarization of carriers at RT. Here situation is far from being satisfactory. For example, for $\mathrm{ZnMnO}$ we observed that as a consequence of nonuniformities of $\mathrm{Mn}$ distribution, photoluminescence mostly comes from regions with low Mn fractions and thus magneto-optical effects are very weak [36]. Definitely magnetic effects
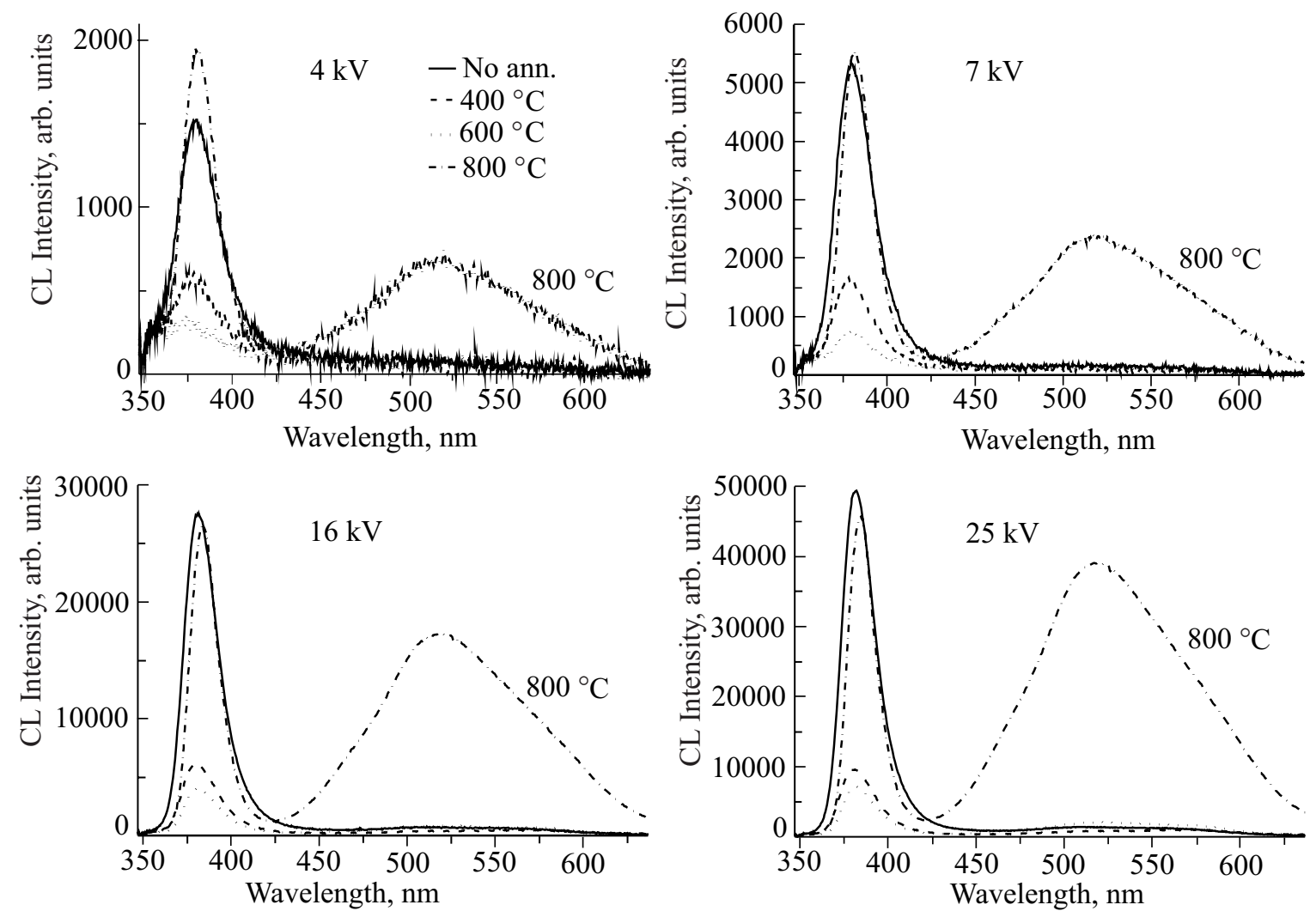

Fig. 4. CL spectra taken at four different accelerating voltages (depth-profiling) for ZnCoO sample as grown and after annealing at 400,600 and $800{ }^{\circ} \mathrm{C}$. 
are very local. On other hand we observed strong polarization of some of the photoluminescence (PL) emissions, as the consequence of spin selection rules for carrier trapping via deep Mn-related level [36,37]. This only contributes to the present misunderstanding of magnetic interactions in TM doped semiconductors.

\section{ZnO for optoelectronic and photovoltaic applications as transparent conductive oxide}

Despite more than 50 years of history, the time which passed from the introduction of the first photovoltaic cell, use of PV devices to harvest the solar energy is still very limited. This is because a payback time for the presently used generations of solar cells is very long-25-30 years, which is comparable to their lifetime. Several solutions are extensively studied at present to increase cells efficiency and reduce their costs [38-40]. To reduce material costs thinner cells are introduced at present (the so-called second generation of the PV cells) or inorganic materials are replaced by organic ones (polymers, the third generation of the PV cells). Another promising approach is to replace relatively expensive ITO (used as transparent electrode) with another cheaper material [11].

$\mathrm{ZnO}$ is one of the most studied materials for the transparent conductive oxide (TCO) application. It is transparent for visible and near infrared light even when heavily $n$-type doped. We demonstrated recently that the $\mathrm{ZnO}$ films grown by the ALD with free electron concentration at RT of $10^{20} \mathrm{~cm}^{-3}[11,41,42]$ are suitable for the application as a transparent electrode in photovoltaic devices and in organic light emitting diodes (OLEDs). Moreover, we demonstrated that such films can replace ITO in practical devices. For example, for the OLED structure based on $\mathrm{Alq}_{3}$ we reached light emission efficiency identical to that achieved in the structure using ITO as the transparent electrode [9].

Further material improvement was achieved by us by doping $\mathrm{ZnO}$ films with $\mathrm{Al}$ [43]. For this we developed a doping procedure in the ALD process, which allowed reduction of resistivity of $\mathrm{ZnO}$ films by one order in magnitude [43]. These films show properties very close to those of ITO. Importantly, the deposition method used by us uses rather inexpensive precursors and the ALD reactors can easily be scaled up allowing substrates of several square meters in size making the recent developments very promising.

\section{Hybrid ZnO/organic material structures for optoelectronic and photovoltaic applications}

Organic materials (e.g., conductive polymers) are intensively studied for applications in electronic, photovoltaic and optoelectronic devices. They show mostly $p$ type conductivity, with electric properties comparable to those of amorphous silicon. A bright light emission was demonstrated for OLEDs, resulting in the introduction of white light emitting panels for an overhead illumination in last months.

These advantageous properties of polymers were known from many years. It took however several years to overcome the main limitation of these materials. It was necessary to solve the problem of their low time stability. They degrade fast upon environment effects, with water vapors being the most destructive ones. In the case of commercialized optoelectronic displays this was avoided by embedding organic films between glass panels. This however is not the most favorable solution.

It was recently demonstrated that coating of polymers with transparent wide band gap materials can improve their time stability $[7,44]$. The first attempts to use $\mathrm{ZnO}$ for such coating were not very encouraging. Much better results were obtained for $\mathrm{TiO}_{2}$ films [44]. Our recent investigations showed however that if the ALD method is used coating with $\mathrm{ZnO}$ can be advantageous.

For deposition on top of organic material a low thermal budget of a growth is required and we must avoid very reactive precursors. Low growth temperature (well below $200{ }^{\circ} \mathrm{C}$ ) and high conformality of $\mathrm{ZnO}$ films grown by the ALD enabled us construction of several devices based on $\mathrm{ZnO}$ /organic material for electronic, optoelectronic and photovoltaic applications $[1,7,9]$.

As already mentioned the first attempts with $\mathrm{ZnO}$ as the coating material were not very promising. Fast water penetration through $\mathrm{ZnO}$ films was related to high vacancy concentration in this material. Here use of the ALD method is very crucial. Once growth conditions were optimized $\mathrm{ZnO}$ films showing only the band edge emission were obtained by us [4-6]. By coating of organic material with such $\mathrm{ZnO}$ films devices stable in time were constructed [7,38] (see Fig. 5). In such devices $\mathrm{ZnO}$ acts not only as the transparent electrode, but also helps to reduce environment effects, stabilizing organic material.

Moreover, junctions of the type $\mathrm{ZnO}$ /organic material show very good electrical properties. High rectification ratio of Schottky junctions for the $\mathrm{P} 3 \mathrm{HT} / \mathrm{ZnO}$ hybrid structure was demonstrated by us [7]. This demonstrates chances for their use in cheap cross-bar memory devices.

\section{Conclusions}

Till recently $\mathrm{ZnO}$ was widely used in chemical, food and cosmetic industry, with a very limited use in electronics, optoelectronics and photovoltaics. This may change soon. Several possible applications of $\mathrm{ZnO}$ are now tested, as discussed in the present work. The ones more advanced relate to the use of $\mathrm{ZnO}$ in transparent electronics and as the TCO material. 

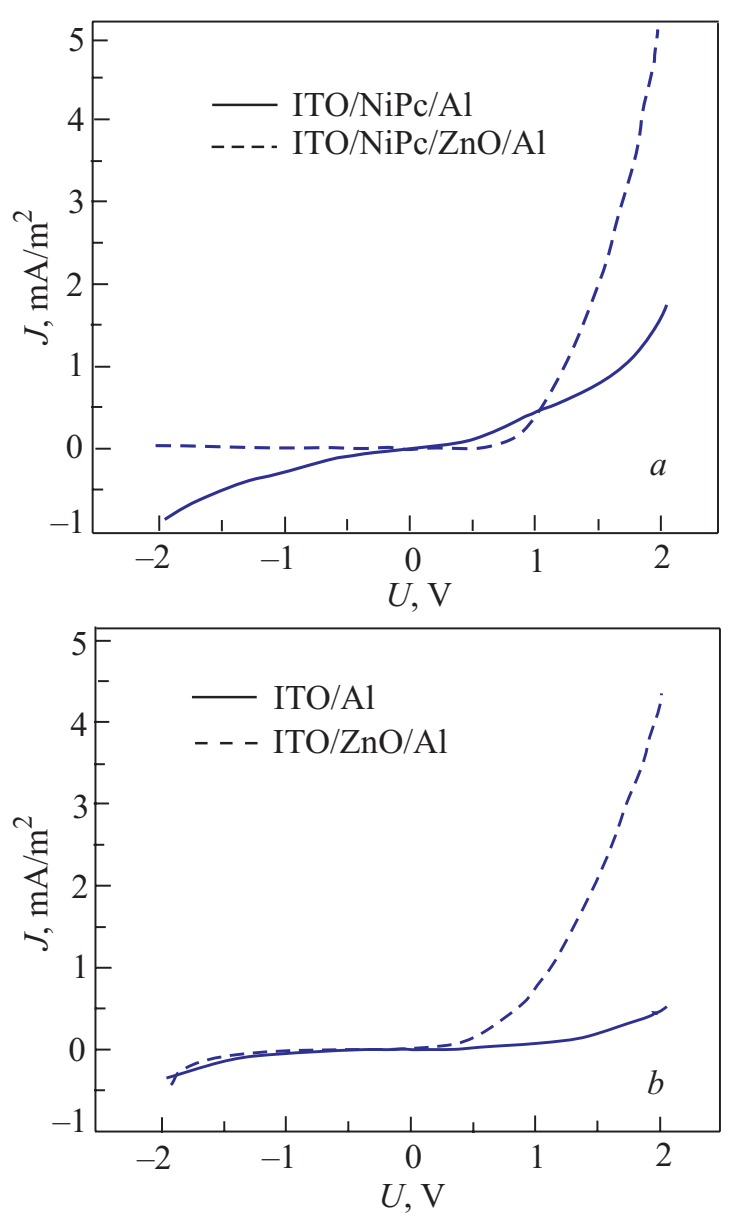

Fig. 5. Comparison of $J(U)$ characteristics measured for hybrid structure of $\mathrm{ZnO}$ with $\mathrm{Ni}$ phthalocyanine $(\mathrm{NiPc})$ for as grown structures (with and without $\mathrm{ZnO}$ coating) (a) and after 30 days of their storage in air $(b)$.

\section{Acknowledgments}

This work was supported in part by the European Union within European Regional Development Fund, through grant Innovative Economy (POIG.01.01.02-00-008/08).

1. M. Godlewski, E. Guziewicz, T. Krajewski, P. Kruszewski, Ł. Wachnicki, K. Kopalko, A. Wójcik, and V. Osinniy, Microelectronic Engineering 85, 2434 (2008).

2. N. Huby, S. Ferrari, E. Guziewicz, M. Godlewski, and V. Osinniy, Appl. Phys. Lett. 92, 023502 (2007).

3. G. Tallarida et al., to be published.

4. T. Krajewski, E. Guziewicz, M. Godlewski, Ł. Wachnicki, I.A. Kowalik, K. Kopalko, A. Wójcik, V. Osinniy, and M. Guziewicz, Microelectron. J. 40, 293 (2009).

5. E. Guziewicz, I.A. Kowalik, M. Godlewski, K. Kopalko, V. Osinniy, A. Wójcik, S. Yatsunenko, E. Łusakowska, and W. Paszkowicz, J. Appl. Phys. 103, 033515 (2008).

6. E. Guziewicz, M. Godlewski, T. Krajewski, Ł. Wachnicki, A. Szczepanik, K. Kopalko, A. Wójcik-Głodowska, E. Przeździecka, W. Paszkowicz, E. Łusakowska, P. Krus- zewski, N. Huby, G. Tallarida, and S. Ferrari, J. Appl. Phys. 105, 122413 (2009).

7. E. Katsia, N. Huby, G. Tallarida, B. Kutrzeba-Kotowska, M. Perego, S. Ferrari, F.C. Krebs, E. Guziewicz, M. Godlewski, and G. Łuka, Appl. Phys. Lett. 94, 143501 (2009).

8. P.Y. Stakhira, G.L. Pakhomov, V.V. Cherpak, D. Volynyuk, G. Luka, M. Godlewski, E. Guziewicz, and Z.Yu. Hotra, Cent. Eur. J. Phys. 8, 798 (2010).

9. M. Godlewski, E. Guziewicz, S. Gierałtowska, G. Łuka, T. Krajewski, Ł. Wachnicki, and K. Kopalko, Acta Phys. Polonica A116, Suppl. S-19 (2009).

10. Semyung Kwon, Seokhwan Bang, Seungjun Lee, Sunyeol Jeon, Wooho Jeong, Hyungchul Kim, Su Cheol Gong, Ho Jung Chang, Hyung-ho Park, and Hyeongtag Jeon, Semicond. Sci. Technol. 24, 035015 (2009).

11. M. Godlewski, E. Guziewicz, G. Łuka, T. Krajewski, M. Łukasiewicz, Ł. Wachnicki, A. Wachnicka, K. Kopalko, A. Sarem, and B. Dalati, Thin Solid Films 518, 1145 (2009).

12. S. Gierałtowska, D. Sztenkiel, E. Guziewicz, M. Godlewski, B.S. Witkowski, Ł. Wachnicki, E. Łusakowska, W. Paszkowicz, R. Minikayev, T. Dietl, and M. Sawicki, Acta Phys. Polonica (in press).

13. S. Datta and B. Das, Appl. Phys. Lett. 56, 665 (1990).

14. T. Dietl, H. Ohno, F. Matsukura, J. Cibert, and D. Ferrand, Science 287, 1019 (2000).

15. K. Ueda, H. Tabata, and T. Kawai, Appl. Phys. Lett. 79, 988 (2001).

16. A. Wolos, M. Palczewska, M. Zajac, J. Gosk, M. Kaminska, A. Twardowski, M. Bockowski, I. Grzegory, and S. Porowski, Phys. Rev. B69, 115210 (2004).

17. M. Godlewski, A. Wójcik-Głodowska, E. Guziewicz, S. Yatsunenko, A. Zakrzewski, Y. Dumont, E. Chikoidze, and M.R. Phillips, Optical Materials 31, 1768 (2009).

18. A. Wójcik, K. Kopalko, M. Godlewski, E. Guziewicz, R. Jakieła, R. Minikayev, and W. Paszkowicz, Appl. Phys. Lett. 89, 051907 (2006).

19. A. Wójcik, M. Godlewski, E. Guziewicz, R. Jakieła, M. Kiecana, M. Sawicki, M. Guziewicz, M. Putkonen, L. Niinistö, Y. Dumont, and N. Keller, Appl. Phys. Lett. 90, 082502 (2007).

20. A. Tomaszewska-Grzęda, A. Opalińska, E. Grzanka, W. Łojkowski, A. Gedanken, M. Godlewski, S. Yatsunenko, V. Osinniy, and T. Story, Appl. Phys. Lett. 89, 242102 (2006).

21. M. Godlewski, A. Wójcik, K. Kopalko, V.Yu. Ivanov, Z. Wilamowski, R. Jakieła, E. Guziewicz, A. Szczepanik, P. Dłużewski, E. Chikoidze, J. Barjon, Y. Dumont, M. Putkonen, L. Niinistö, and Dong Tang, Acta Physica Polonica (a) 112, 261 (2007).

22. M. Łukasiewicz, B. Witkowski, M. Godlewski, E. Guziewicz, M. Sawicki, W. Paszkowicz, E. Łusakowska, R. Jakieła, T. Krajewski, I.A. Kowalik, and B.J. Kowalski, Acta Physica Polonica 116, 1111 (2009).

23. M. Godlewski, E.M. Goldys, M.R. Phillips, R. Langer, and A. Barski, J. Mater. Res. 15, 495 (2000). 
24. A. Ney, K. Ollefs, S. Ye, T. Kammermeier, V. Ney, T.C. Kaspar, S.A. Chambers, F. Wilhelm, and A. Rogalev, Phys. Rev. Lett. 100, 157201 (2008).

25. J.M.D. Coey, K. Wongsaprom, J. Alaria, M. Venkatesan, J. Phys. D: Appl. Phys. 41, 134012 (2008).

26. A.J. Behan, A. Mokhtari, H.J. Blythe, D. Score, X.-H. Xu, J.R. Neal, A.M. Fox, and G.A. Gehring, Phys. Rev. Lett. 100, 047206 (2008).

27. J.H. Park, M.G. Kim, H.M. Jang, S. Ryu, and Y.M. Kim, Appl. Phys. Lett. 84, 1338 (2004).

28. A. Bonanni and T. Dietl, Chem. Soc. Rev. 39, 528 (2010).

29. T. Dietl, T. Andrearczyk, A. Lipińska, M. Kiecana, Maureen Tay, and Yihong Wu, Phys. Rev. B76, 155312 (2007).

30. X.J. Liu, C. Song, F. Zeng, X.B. Wang, and F. Pan, J. Phys. D: Appl. Phys. 40, 1608 (2007).

31. D. Karmakar, S.K. Mandal, R.M. Kadam, P.L. Paulose, A.K. Rajarajan, T.K. Nath, A.K. Das, I. Dasgupta, and G.P. Das, Phys. Rev. B75, 144404 (2007).

32. D. Iusan, B. Sanyal, and O. Eriksson, Phys. Rev. B74, 235208 (2006).

33. Q. Wang, Q. Sun, P. Jena, and Y. Kawazoe, Phys. Rev. B79, 115407 (2009).

34. C.H. Park and D.J. Chadi, Phys. Rev. Lett. 94, 127204 (2005).

35. M. Łukasiewicz et al., to be published.

36. M. Godlewski, A. Wąsiakowski, V.Yu. Ivanov, A. WójcikGłodowska, M. Łukasiewicz, E. Guziewicz, R. Jakieła, K.
Kopalko, and A. Zakrzewski, Optical Materials 32, 680 (2010).

37. V.Yu. Ivanov, M. Godlewski, S. Yatsunenko, A. Khachapuridze, Z. Golacki, M. Sawicki, A. Omel'chuk, M. Bulany, and A. Gorban', Phys. Status Solidi (c) 1, 250 (2004).

38. Survey of Energy Resources, World Energy Council (2007) (www.worldenergy.org/publications/survey_of_energy_resources_2007).

39. B. Späth, J. Fritsche, F. Säuberlich, A. Klein, and W. Jaegermann, This Solid Films 480-481, 204 (2005).

40. O. Bamiduro, H. Mustafa, R. Mundle, R.B. Konda, and A.K. Pradhan, Appl. Phys. Lett. 90, 252108 (2007).

41. A. Szczepanik, Ł. Wachnicki, M. Godlewski, E. Guziewicz, K. Kopalko, E. Janik, E. Łusakowska, A. Czerwiński, M. Płuska, and S.A. Yatsunenko, J. Phys: Conf. Ser. 146, 012017 (2009).

42. E. Janik, A. Wachnicka, Ł. Wachnicki, E. Guziewicz, M. Godlewski, S. Kret, W. Zaleszczyk, E. Dynowska, A. Presz, G. Karczewski, and T. Wojtowicz, Nanotechnology 21, 015302 (2010).

43. G. Łuka, T. Krajewski, Ł. Wachnicki, K. Kopalko, E. Łusakowska, W. Paszkowicz, E. Guziewicz, and M. Godlewski, Phys. Status Solidi (a) 207, 1568 (2010).

44. F.C. Krebs, Solar Energy Materials \& Solar Cells 92, 715 (2008). 